\title{
Consistency of the Ether Theory with the Time Concept, the Mass Concept and the Special Relativity
}

\author{
David Zareski ${ }^{1,2}$ \\ ${ }^{1}$ I.A.I, Israel Aerospace Industries, Yehud Israel \\ ${ }^{2}$ Université Paul Sabatier, Toulouse, France \\ Correspondence: David Zareski, I.A.I, Israel Aerospace Industries, Yehud Israel; Université Paul Sabatier, \\ Toulouse, France. E-mail: zareski@inter.net.il \\ Received: June 21, 2020 \\ Accepted: July 10, 2020 \\ Online Published: July 31, 2020 \\ doi:10.5539/apr.v12n4p1 \\ URL: http://dx.doi.org/10.5539/apr.v12n4p1
}

\begin{abstract}
We demonstrate that it is by considering the existence of an "ether" that one can define the notions of time, velocity and mass, and prove that Einstein's second principle implies the existence of this ether since the light propagates independently of its emitter velocity. Then, that the particle velocity is a group velocity different from the phase velocity when the particle is massive. One interprets then the Morley-Michelson experiment in the frame of the ether.

Let be $L(0)$ a fixed distance between two points immobile in a long vehicle in the ether, and let a photon that, when this vehicle is immobile relatively to the ether, travels the distance $L(0)$ in this vehicle. An observer immobile in the immobile vehicle sees that the distance traveled by the photon is also $L(0)$. But, if the vehicle moves, e.g., at the velocity $V$ relatively to the ether, and the photon at the velocity $c$ also relatively to the ether, then an observer immobile in the moving vehicle will find that the distance traveled by this photon is not $L(0)$ but is $L(V)$ defined by

$L(V)=L(0) \sqrt{1-(V / c)^{2}}$. We prove then that $L(V)$ is not the new dimension of the vehicle, but is simply the distance travelled by the photon in this vehicle. That is, as demonstrated in our previous publications: the ether volumetric density is constant.
\end{abstract}

Résumé. Nous démontrons que c'est en considérant l'existence de l'“éther" qu'on peut définir la notion de temps, de vitesse et de masse et prouver que le second principe d'Einstein implique l'existence de cet éther puisque la lumière s'y propage indépendamment de la vitesse de l'émetteur. Puis que la vitesse de la particule est une vitesse de groupe qui est différente de la vitesse de phase lorsque cette particule est massive. Nous interprétons ensuite l'expérience de Morley-Michelson dans le cadre de l'éther.

Soit $L(0)$ une distance fixe entre deux points immobiles dans un long véhicule dans l'ether et soit un photon qui, lorsque ce véhicule est immobile relativement à l'éther, parcourt la distance $L(0)$ dans ce véhicule. Un observateur immobile dans ce véhicule immobile verra que la distance parcourue par ce photon est aussi $L(0)$. Mais si ce véhicule se déplace, par exemple, à la vitesse $V$ relativement à l'ether, et ce photon à la vitesse $c$ relativement à l'éther, un observateur immobile dans ce véhicule mobile trouvera que la distance parcourue par ce photon ne sera pas $L(0)$ mais sera $L(V)$ définie par $\left.L(V)=L(0) \sqrt{1-(V / c)^{2}}\right)$. Nous prouvons, que $L(V)$

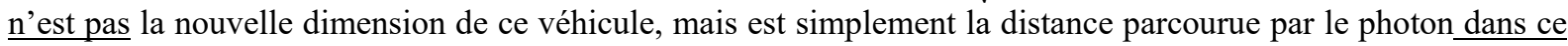
vehicule. Ainsi que nous l'avons démontré dans nos précédentes publications, la densité de l'éther reste constante.

Keywords: Special Relativity, Ether, Time, Mass, Group and Phase Velocities, Fictive Length Change of the Moving Rod

\section{Introduction}

The concept of ether envisaged in particular by Maxwell and Einstein (Refs. 1-5) has been developed by the present author in previous articles, Cf. Refs. 6-9. This contribution is unique and original, as proven by the following brief and limited survey of recent contributions on the same subject of the ether and its properties. In his paper "Motion Trough the Ether", Silvertooth (Ref. 10) accepts the existence of the ether, but also affirms to have disproved the Principle of Relativity. In the present paper I demonstrate that the Principle of Relativity is logically equivalent to the existence of the ether. In another paper "Experimental Evidence of the Ether-dragging Hypothesis in GPS 
Data", Sato (Ref. 11) considers that there is an ether drag. In this paper I will prove that there is no drag of the ether but that a particle is a single particle wave $\hat{\xi}$ that is, it is a superposition of waves $\xi$ that creates a vibrating globule of small volume of the ether. In his paper "The Relative Motion of the Earth and the Ether Detected", Gift (Ref. 12) confirms the existence of a detectable ether, but also affirms that the existence of the ether is inconsistent with the Special Relativity. Again, I prove in this present paper that the Principle of Relativity is logically equivalent to the existence of the ether. I could continue with the survey of the vast literature on the ether, but I do not deem that it is necessary.

In the present paper we show first that, contrarily to some opinions, one can define the time. In particular one can define it when one considers the ether which is, Cf. Ref. 6-9, an elastic isotropic medium of constant properties, in which when it is free, the light is propagated always at the same manner independently of the emitter. Note: we used the term "manner" instate of "velocity" because the notion of velocity of propagation in the ether is defined here below. It is this constancy of the light propagation that permits to define the unity of time. Indeed, a unity of time can be defined as to be proportional to a given length in the ether traveled by a photon, for example, one second will be proportional to the distance of numerical value $c$ travelled by the photon in the free ether. The velocity of the light, i.e., of the photon, is therefore $c$ which is constant in the free ether, and we can define the velocity $V$ and the mass of a massive particle.

It appears as we show that the Special Relativity is based on the hypothesis that it exists the ether. Now for the photon which is a massless particle the group and the phase velocities are identical, but these two kinds of velocity are different for massive particle. Furthermore, it appears, as we show, that a continuation of Einstein's special relativity theory, permits to demonstrate the results of the Morley-Michelson experiment. Then, that in this experiment there is not any change of the ether density, but only a change of the distance travelled by the photon in the moving vehicle.

\section{Time Definition and Vibration Propagation in an Elastic Medium, the "Ether"}

Let us consider an elastic isotropic medium of constant properties not depending upon the location in that medium. Since it is elastic, the effects due to an external cause that perturbs even its a small volume, are propagated there. We call "ether" such a medium.

If $\delta \ell$ denotes a given constant length in the ether, and $c$ a given constant parameter defined in the following, then, we call unity of time duration and denote it $\delta$ t, the quantity defined by

$$
\delta t \equiv \delta \ell / c
$$

If $\Delta \ell$ denotes a length, one defines the time duration for the light to travel the distance $\Delta \ell$ and denotes it $\Delta t$, the number of $\mathrm{c}$ contained in $\Delta \ell$, that is,

$$
\Delta t=\Delta \ell / c \equiv \delta t \Delta \ell / \delta \ell
$$

The time duration $\Delta t$ and the length $\Delta \ell$ can be denoted more explicitly

$$
\begin{aligned}
\Delta t & =t-t_{0} \\
\Delta \ell & =\ell-\ell_{0}
\end{aligned}
$$

from (2), (3), and (4), it follows that

$$
t-t_{0}=\delta t\left(\ell-\ell_{0}\right) / \delta \ell
$$

Having defined the time duration $\Delta t$, the instant $t$ is defined by

$$
t=\ell / c \equiv \delta t \ell / \delta \ell
$$

that is, $t$ denotes the number of $c$ contained in the length $\ell$. It appears that $c$ defined by

$$
c=\Delta \ell / \Delta t \equiv \delta \ell / \delta t \equiv \ell / t
$$

denotes the propagation velocity in the free ether of a perturbations called "light" created even in a small volume of the ether. The light is in the form of sinusoidal waves, of which their superpositions of close frequencies forms globules called "photons" that moves also at the velocity $c$. It follows that:

The existence of the time is equivalent to the existence of the elastic isotropic homogeneous medium called ether.

Indeed, the existence of the time implies that of the ether, since in the absence of the ether, waves would 
not be propagated always with the same velocity, therefore one could not define the time as being proportional to a length propagated by such waves. Reciprocally, the existence of the ether permits to define the time, since if one could not define the time for example taken by a wave to travel a given distance, one could not define the ether since a time duration is proportional to a length in the ether.

In that context where the velocity propagation in the free ether is the light velocity $c$, let us consider Einstein's principle $P_{2}$ stated as following, Cf. pp 41, Ref. 1:

"Any ray of light moves in the stationary system of coordinates with the determined velocity $c$, whether the ray is emitted by a stationary or a moving system."

In fact, this principle implies the existence of an ether as here above defined. Indeed, in the absence of any elastic medium, waves could not be propagated, but only of entities of which the velocities would not be the same and independent of those due to the emitters and of other causes that could move in the empty space.

Numerus authors preconized the existence of the ether, in particular, Maxwell and Einstein. Maxwell wrote, in Art. 866 of Ref. 3:

"...Hence all these theories lead to the conception of a medium in which the propagation takes place, and if we admit this medium as an hypothesis, I think it ought to occupy a prominent place in our investigations."

and Einstein in Ref. 4:

"...Recapitulating, we may say that according to the general theory of relativity space is endowed with physical qualities; in this sense, therefore, there exists an ether. According to the general theory of relativity space without ether is unthinkable; for in such space there not only would be no propagation of light, but also no possibility of existence for standards of space and time (measuring-rods and clocks), nor therefore any space-time intervals in the physical sense...... “. See also Ref. 5.

It appears again that the stationary system cited by Einstein, i.e., the ether, is an elastic isotropic homogenous medium, since light is propagated there at the velocity $c$ independently of its emitter velocity. if there were no such a medium, the velocity of an emitted signal would depend upon its emitter.

\section{Definition of the Particle Mass and of the Velocity of Massive Particle}

A particle of kinetic energy $h v$ is said to have the mass $m$ and denoted $\operatorname{Par}\left(m c^{2}+h v\right)$ if, for example, being free, then during the time $t$ during with the photon i.e., the $\operatorname{Par}(0+h v)$ travels the distance $t c$, this $\operatorname{Par}\left(m c^{2}+h v\right)$ travels the distance $t c \sqrt{1-\left[m c^{2} /\left(m c^{2}+h v\right)\right]^{2}}$. That is to say that the mass $m$ causes to the free $\operatorname{Par}\left(m c^{2}+h v\right)$ to move at the velocity $V$ for which the expression is

$$
V=c \sqrt{1-\left[m c^{2} /\left(m c^{2}+h v\right)\right]^{2}}
$$

instead of moving at the velocity $c$ of the free $\operatorname{Par}(0+h v)$. Reciprocally, $m$ being defined by

$$
m=\frac{h v}{c^{2}} \frac{\sqrt{c^{2}-V^{2}}}{\left(c-\sqrt{c^{2}-V^{2}}\right)}
$$

it follows that: $[V=0] \Leftrightarrow[m=\infty]$ and $[V=c] \Leftrightarrow[m=0]$.

\section{Group Velocity and Phase Velocity}

Having defined the notion of time in the ether, one presents now another definition of the velocity of a particle of mass $m$. By saying that $\operatorname{Par}\left(m c^{2}+h v\right)$ moves at the velocity $V$, we mean that: during the time duration $\Delta t$ where $\operatorname{Par}\left(m c^{2}+h v\right)$ covers the distance $\Delta \ell_{\left(m c^{2}+h v\right)}$, a $\operatorname{Par}(0+h v)$ covers the distance $\Delta \ell_{(0+h v)}$, that is,

$$
\Delta t=\Delta \ell_{(0+h v)} / c
$$

it follows that

$$
V=\frac{\Delta \ell_{\left(m c^{2}+h v\right)}}{\Delta t}=\frac{\Delta \ell_{\left(m c^{2}+h v\right)}}{\Delta \ell_{(0+h v)}} \cdot c
$$

Now, to the velocity $V$ of a $\operatorname{Par}\left(m c^{2}+h v\right)$ is associated the phase velocity $V_{P}$ by the relation, Cf. Eq. (3.43), of Ref. 7,

$$
\frac{\partial}{\partial E_{T}}\left(\frac{E_{T}}{V_{P}}\right)=\frac{1}{V}
$$


This relation is always valid even for the photon since its velocity does not depend upon its energy. Now, since $V_{P}$ is the phase velocity, $V$ is the group velocity that is a superposition of waves of phase velocity $V_{P}$ it follows, Cf. e.g., Eq. (5.7) of Ref. 7, that to the $\operatorname{Par}\left(m c^{2}+h v\right)$ of velocity $V$ and of phase velocity $V_{P}$, is associated the particle wave $\widehat{\boldsymbol{\xi}}_{P a r}\left(m c^{2}+h v\right)$ for which the expression is

$$
\widehat{\boldsymbol{\xi}}_{P a r\left(m c^{2}+h v\right)}=\xi_{0} \exp \left[i \omega\left(-t+\int \frac{d l}{V_{P}}\right)\right] \operatorname{SINC}\left[\frac{\Delta \omega}{2}\left(-t+\int \frac{d l}{V}\right)\right]
$$

Where: $\omega \equiv E_{T} / \hbar \equiv\left(m c^{2}+h v\right) / \hbar$ and $\operatorname{SINC}(x) \equiv(\sin x) / x$.

To the $\operatorname{Par}(0+h v)$ is associate the wave $\widehat{\boldsymbol{\xi}}_{\operatorname{Par}(0+h v)}$ for which the expression is

$$
\widehat{\boldsymbol{\xi}}_{\text {Par }(0+h v)}=\xi_{0} \exp \left[i 2 \pi v\left(-t+\frac{l}{c_{p}}\right)\right] \operatorname{SINC}\left[\frac{\Delta \omega}{2}\left(-t+\frac{l}{c_{p}}\right)\right]
$$

where $c_{p}$ denotes the photon velocity even in a perturbed ether.

\section{Recalls on the Morley-Michelson Experiment}

One recalls: that in the Morley-Michelson experiment, the two photons of different trajectories meet, Cf. Eqs. (36)(40) of Ref. 9, that, in the perpendicular trajectory, the time $t_{\perp}$ taken by the photon to return at its depart point in the vehicle is

$$
t_{\perp}=2 L / \sqrt{c^{2}-V^{2}}
$$

and that, in the parallel trajectory, the time $t_{x}$ taken by the photon to return at its depart point in the vehicle is

$$
t_{x}=2 c L_{x} /\left(c^{2}-V^{2}\right)
$$

Since these two photons meet, it follows that

$$
t_{\perp}=t_{x}
$$

that is,

$$
L_{x}=L \sqrt{\left(1-V^{2} / c^{2}\right)}
$$

Using the notation $L(0)$ instead of $L$, and $L(V)$ instead of $L_{x}$, one has, Cf. also Eq. (32) of Ref. 9,

$$
L(V)=L(0) \sqrt{1-(V / c)^{2}}
$$

\section{The Results of the Morley-Michelson Experiment Explained by the Special Relativity}

Let $\boldsymbol{e}_{i},(\mathrm{i}=1,2,3)$, denote an orthonormal system of coordinates $K$ immobile in the ether, $P(\boldsymbol{V})$ a point moving there at the velocity $\boldsymbol{V}$, i.e., immobile in a vehicle of velocity $\boldsymbol{V}$ relatively to the ether. An observer $O b[P(\boldsymbol{V})]$ located at $P(\boldsymbol{V})$ sees that a photon moving in the same direction as $\boldsymbol{V}$ is of velocity $\boldsymbol{V}_{\boldsymbol{p h}+}$ such that

$$
\boldsymbol{V}_{p h+}=\boldsymbol{c}-\boldsymbol{V}
$$

while a photon moving in the opposite direction $-\boldsymbol{V}$ is seen by this $O b[P(\boldsymbol{V})]$, to be of velocity $\boldsymbol{V}_{\boldsymbol{p h}-}$ such that

$$
\boldsymbol{V}_{p h-}=-\boldsymbol{c}-\boldsymbol{V}
$$

In this vehicle, one considers a photon located at $P(\boldsymbol{V})$ at the time $t_{0}$ that moves in the same direction as $\boldsymbol{V}$ and, after having covered a distance $x^{\prime}$ in this vehicle, is reflected and returns at its departure point $P(\boldsymbol{V})$ in this vehicle. Let $\tau$ denote the time duration measured by a watch fixed at $P(\boldsymbol{V})$, i.e., fixed in this vehicle, taken by the photon to cover the distance $x^{\prime}$ in the direction of $\boldsymbol{V}$ in the vehicle, and to return at its departure point $P(\boldsymbol{V})$ of which the distance is also $x^{\prime}$ in this vehicle. As defined in page 44 from the line 14 of Ref 1 , this duration $\tau$ is, in particular, a function of $\frac{x^{\prime}}{c-V}$ and of $\frac{x^{\prime}}{c+V}$. Now let us consider more profoundly the physical significance of these quantities. In that context, we show now that:

$$
\frac{x^{\prime}}{c-V}=\frac{x^{\prime}+V \Delta t_{1}}{c}=\Delta t_{1} \text { and } \frac{x^{\prime}}{c+V}=\frac{x^{\prime}-V \Delta t_{2}}{c}=\Delta t_{2}
$$

where $\Delta t_{1}$ Resp. $\Delta t_{2}$ denotes the time, measured by a clock immobile in $K$, that takes the photon to travel the 
distance $x^{\prime}$ measured in the vehicle in the direction of its motion, Resp. in the opposite direction. Since, as one can verify,

$$
\frac{x^{\prime}}{c-V}-\frac{x^{\prime}+V \Delta t_{1}}{c}=\frac{V}{c}\left(\frac{x^{\prime}}{c-V}-\Delta t_{1}\right)
$$

one has the following equivalence:

$$
\frac{x^{\prime}}{c-V}=\Delta t_{1} \Leftrightarrow \frac{x^{\prime}}{c-V}=\frac{x^{\prime}+V \Delta t_{1}}{c}
$$

and in the same way, one has the following equivalence

$$
\frac{x^{\prime}}{c+V}=\Delta t_{2} \Leftrightarrow \frac{x^{\prime}}{c+V}=\frac{x^{\prime}-V \Delta t_{2}}{c}
$$

Now these terms $\frac{x^{\prime}}{c-V}$ and $\frac{x^{\prime}}{c+V}$ appear in Einstein's equation PP. 44, of Ref. 1, namely,

$$
\tau\left(0, t_{0}\right)+\tau\left(0, t_{0}+\frac{x^{\prime}}{c-V}+\frac{x^{\prime}}{c+V}\right)=2 \tau\left(x^{\prime}, t_{0}+\frac{x^{\prime}}{c-V}\right)
$$

for which the solution is

$$
\tau=\sqrt{1-(V / c)^{2}}\left(t-\frac{V x^{\prime}}{c^{2}-V^{2}}\right)
$$

Note that we repeated Einstein's reasoning of PP. 44 Ref. 1, and of the first equation of page 45 there, in order to obtain Eq. (26).

Now the constancy of $x^{\prime}$ in the vehicle that moves at the velocity $\boldsymbol{V}$ relatively to the ether is the fundamental fact in this reasoning. Indeed, $t$ that appears in Eq. (26) is a parameter that has to be defined in order to define completely $\tau$. To this purpose, we are going to define $t$ in such a way that Eq. (26) will become identical to Eq. (15), that is to say that one wants to determine $t$ such that (26) will become

$$
\tau=2 x^{\prime} / \sqrt{c^{2}-V^{2}}
$$

which is of the same form as Eq. (15), where $\tau$ is no other than $t_{\perp}$ and $x^{\prime}$ is no other than $L$. Now, in order to determine the expression for $t$ such that (26) will becomes (27), one has to determine $t$ as a solution of the equation

$$
\sqrt{1-(V / c)^{2}}\left(t-\frac{V x^{\prime}}{c^{2}-V^{2}}\right)=\frac{2 x^{\prime}}{\sqrt{c^{2}-V^{2}}}
$$

The solution of this equation, that is, the expression for $t$ such that (26) becomes (27), is:

$$
t=x^{\prime}(2 c+V) /\left(c^{2}-V^{2}\right)
$$

That is, by inserting this expression for $t$ into Eq. (26), one obtains Eq. (27) which is identical with the result (15) of the Morley-Michelson experiment where $\tau \equiv t_{\perp}$ and $x^{\prime} \equiv L$. It follows that:

the special relativity explains the results of the Morley-Michelson experiment.

\section{Interpretation of the Morley-Michelson Experiment Results in the Frame of the Ether Theory}

It follows from the Morley-Michelson experiment results, that the distance $L(0)$ travelled by the photon when this length is immobile relatively to the ether, becomes $L(V)$ defined by (19) when this length is, e.g., in a vehicle that moves at the velocity $V$ relatively to the ether in which the photon moves at the velocity $c$ relatively to th ether. One recalls that from Eq. (27) which is the same as Eqs. (15) and (16), one obtains Eq. (19) that defines the change of the length of the trajectory traveled by the photon in the vehicle that moves at the velocity $V$ relatively to the ether. The question is now:

what represents this change of length from $L(0)$ to $L(V)$ that appears in Eq. (19), in other words: this length that changes is the length of what?

Remark. One considers that $L(0)$ is also the material length of the vehicle measured when it is immobile relatively to the ether. 
In order to respond to this question one as to remark that, as demonstrated here above, the photon travels the distance $L(V)$ defined in (19) in this vehicle that moves at the velocity $V$ relatively to the ether. It appears that there are two eventual responses to the question regarding the changes of the length of the vehicle from $L(0)$ to $L(V)$. These two eventualities are the followings:

Eventuality 1: the length $L(0)$ of the vehicle is physically modified and becomes $L(V)$, i.e., becomes the length traveled by the photon in this vehicle;

Eventuality 2: the length $L(0)$ of the vehicle remains unchanged, but the photon travels in this vehicle the distance $L(V)$.

In order to answer to this question, that is, to demonstrate what represents the length $L(V)$ in this vehicle, let us consider the particular case where the vehicle moves at the light velocity $c$, i.e., $V=c$, in this case:

In the Eventuality 1, the distance $L(V)$ becomes $L(c)=0$, Cf. (19). That is to say that, on one hand, the length of the vehicle becomes zero but contains the photon and also the passengers immobile in this vehicle. But on the second hand, these passengers see that they have the same dimensions as they had when they were immobile relatively to the ether, since this hypothetic change of the length should be seen only by observers immobile in the ether, i.e., out of this vehicle.

In the Eventuality 2, the photon travels also the distance 0 in this vehicle, i.e., remains immobile there, but the length of this vehicle seen by an observer immobile in the ether, or seen by an observer immobile in this moving vehicle, remains unchanged at its value $L(0)$, since every point of this vehicle moves at the same velocity. Furthermore, since every point of the vehicle moves at the same velocity, there cannot be any length contraction since if there where a contraction certain points of this vehicle would have to move at a velocity different from $c$.

It appears therefore that the Eventuality 1 is unrealistic since a length is seen at the same time to be zero by an immobile observer in the ether, and to to be $L(0)$ by the passenger in this vehicle. While the Eventuality 2 is realistic, since it is obvious that if the vehicle moves at the velocity $c$ then the photon remains immobile in this vehicle and a passenger immobile in this vehicle or immobile in the ether, sees that the length of this vehicle remains $L(0)$ since every point in this vehicle moves at the same velocity $\boldsymbol{c}$. Finally, it appears that:

\section{$L(V)$ defined in (19), denotes the length traveled by the photon in the vehicle that moves at the velocity $V$ relatively to the ether while the length of this vehicle remains unchanged at the value $L(0)$ that it has when it was immobile relatively to the ether.}

From this fact, it follows the important conclusion relative to the ether that confirms our anterior demonstrations concerning the ether namely:

as shown in our previous publications, it appears again that the ether density remains unchanged.

\section{Conclusions}

Contrarily to what is written by some authors we demonstrate that one can define the time univocally. For example, to be proportional to the distance travelled by the light divided by a known constant parameter, for example $c$, called light velocity. Now, since the light is in the form of waves propagated in the free space always at the same velocity $c$, it must exist an "elastic" medium in which these waves are propagated since, if there were not such an elastic medium then: or there were not waves propagating there or if there were waves propagating there, they would not be propagated at a velocity independently of the emitter. The existence of such an elastic isotropic homogenous medium, i.e., the ether, was preconized by Maxwell, Einstein, other physicists, and developed by the author.

It is the notions of ether and of propagation of waves, i.e., of light there, that permit to define the time and reciprocally it is the notion of time that permits to define the ether. Indeed, the time can be defined as to be proportional to the distance of vibration propagation in the ether, and reciprocally the distance can be defined as to be proportional to the time taken by the photon to cover this distance.

Let us consider a vehicle of length $L(0)$ measured when it is immobile relatively to the ether and an observer that sees a free photon that traveled the distance $L(0)$ in this immobile vehicle. Now, when this vehicle moves at the velocity $V$ relatively to the ether, this observer immobile in the moving vehicle sees that the distance traveled by this photon is not $L(0)$ but is

$L(V)=L(0) \sqrt{1-(V / c)^{2}}$. But one has to remark that $L(V)$ is not the new dimension of the vehicle, but is the distance travelled by the photon in the vehicle. For example if the vehicle would move at the velocity $c$ relatively to the ether, the photon would be seen to be immobile by a passenger immobile in this vehicle since $L(c)=0$. It follows that, as we have demonstrate also in our previous publications the ether density remains constant. 


\section{Conflict of interests}

The authors declare that there is no conflict of interests regarding the publication of this paper.

\section{References}

E. W. Silvertooth, E. W. (1989). Motion Trough the Ether. Silvertooth_Elect, 95(1639), 437-438.

Einstein, A. (1920). Ether and the theory of relativity. The collected papers of Albert Einstein (Springer, Berlin, 1920), address delivered on May 5,1920, at the university of Leyden.

Einstein, A. (1950). On the generalized theory of gravitation. Sci. Am. Spec. Issue, 40-45. https://doi.org/10.1038/scientificamerican0450-13

Einstein, A. (n. d.). On the electrodynamics of moving bodies. Published in Ref. 2.

Gift, S. (2006). The Relative Motion of the Earth and the Ether Detected. Journal of Scientifid Exploration, 20(2).

Lorentz, H. A., Weyl, H., Minkowski, H., \& Einstein, A. (1952). The principle of relativity. Dover, New-York.

Maxwell, J. C. (1954). A Treatise on Electricity and Magnetism. Dover, New-York, 1954.

Sato, M. (2010). Experimental Evidence of the Ether-dragging Hypothesis in GPS Data. Physics Essays, 23(1). https://doi.org/10.4006/1.3301423

Zareski, D. (2001). The elastic interpretation of electrodynamics. Foundation of Physics, 14(5). https://doi.org/10.1023/A:1015513504377

Zareski, D. (2012). On the elasto-undulatory interpretation of fields and particles. Physics Essays, $25,2$. https://doi.org/10.4006/0836-1398-25.2.268

Zareski, D. (2014). Unification of physics by the ether elasticity theory. Global Journal of Science Frontier Research: A Physics and Space Science, 14(6), 1-11. https://doi.org/10.5539/apr.v6n4p48

Zareski, D. (2015). Unification of Physics by the Ether Elasticity Theory (p. 102). ISNB: 978-3-659-67720-5 published by LAP LAMBERT Academic Publishing (2015).

\section{Copyrights}

Copyright for this article is retained by the author(s), with first publication rights granted to the journal.

This is an open-access article distributed under the terms and conditions of the Creative Commons Attribution license (http://creativecommons.org/licenses/by/4.0/). 